\title{
Estudio de las diferencias en la encarnación física de los patrones generadores de lugar entre lo sacro y lo secular en la arquitectura
}

\author{
A study of differences in the physical embodiment of place-making patterns between the sacred and \\ secular in architecture
}

Arsenio T. Rodrigues

https://doi.org/10.17979/aarc.2011.2.2.5066

Lo sagrado es principalmente lo Real. Una Realidad totalmente ajena que no pertenece a este mundo, a pesar de que se manifiesta en él y a través de él. (Mircea Eliade, 1959)

\section{INTRODUCCIÓN}

Definir lo sacro resulta impropio, porque las definiciones conllevan límites y no son inclusivas ${ }^{1}$. No obstante, a pesar de que no se puede definir lo sacro, puede ser sentido y comprendido parcialmente, y su presencia puede conocerse ${ }^{2}$. De acuerdo con el famoso historiador de la religión, Mircea Eliade, lo sacro puede concebirse como «lo opuesto a lo profano», algo que se revela y manifiesta como un orden totalmente diferente a lo profano, haciéndonos saber así de su realidad ${ }^{3}$. Para poder entender lo sacro en la arquitectura, por tanto, tenemos que explorar las múltiples maneras en las que hallamos su inclusión en la creación de lugares (es decir, en la acción de crear un lugar o en la sensación de lugar).

Según Eliade 4 , la desacralización del lugar ha hecho cada vez más difícil para las sociedades modernas el redescubrir las dimensiones existenciales de lo sagrado que una vez fueron fácilmente accesibles a las personas de las sociedades arcaicas en los lugares habituales. En un tiempo dominado por los estilos arquitectónicos insulsos y las estrategias de construcción orientadas a la economía, el redescubrimiento de los principios de diseño y los valores intemporales que han guiado e informado la construcción de lugares desde tiempos inmemoriales, a menudo se descuida o -incluso peorse olvida. Por ello, la motivación que subyace en este estudio es la necesidad de reconectar con dichos principios arquetípicos, y así, reforzar el proceso corpóreo de la arquitectura mundana.

Diversos autores han debatido y desarrollado características o patrones asociados a la creación de lugares en sus respectivas obras. Entre ellos están (en orden ascendente de fecha de publicación): 1) Marcus Vitruvius Pollio en el siglo I a.C., Los diez libros de la arquitectura $^{5}$; 2) Andrea Palladio en 1570 , Los cuatro libros de la arquitectura ${ }^{6}$; 3) Mircea Eliade en 1959, Lo sagrado y lo profano ${ }^{7}$; 4) Christopher Alexander, Sara Ishikawa y Murray Silverstein en 1977, A Pattern Language: Towns, Buildings, Construction ${ }^{8}$; 5) Michael Brill en 1985, Using the Place-Creation Myth to Develop Design Guidelines for Sacred Space9; 6) Charles Moore y Donlyn Lyndon en 1994, Chambers of a Memory Palace10; 7) Phillip Tabb en 1996, Sacred Place: The Presence of Archetypal Patterns in Place Creation ${ }^{11}$; y 8) Christopher Alexander en 2002, The Nature of Order: The Phenomenon of Life ${ }^{12}$. El objetivo de este estudio es promover un interés renovado en el significado y uso de los patrones específicos de creación de lugares que contribuyen a la sacralidad de un lugar.

Los patrones se refieren sobre todo a las características recurrentes o subyacentes que, en primer lugar, 
Fig. 1. Philip Johnson, Howard Barnstone y Eugene Aubry, Rothko Chapel, Houston, 1971.
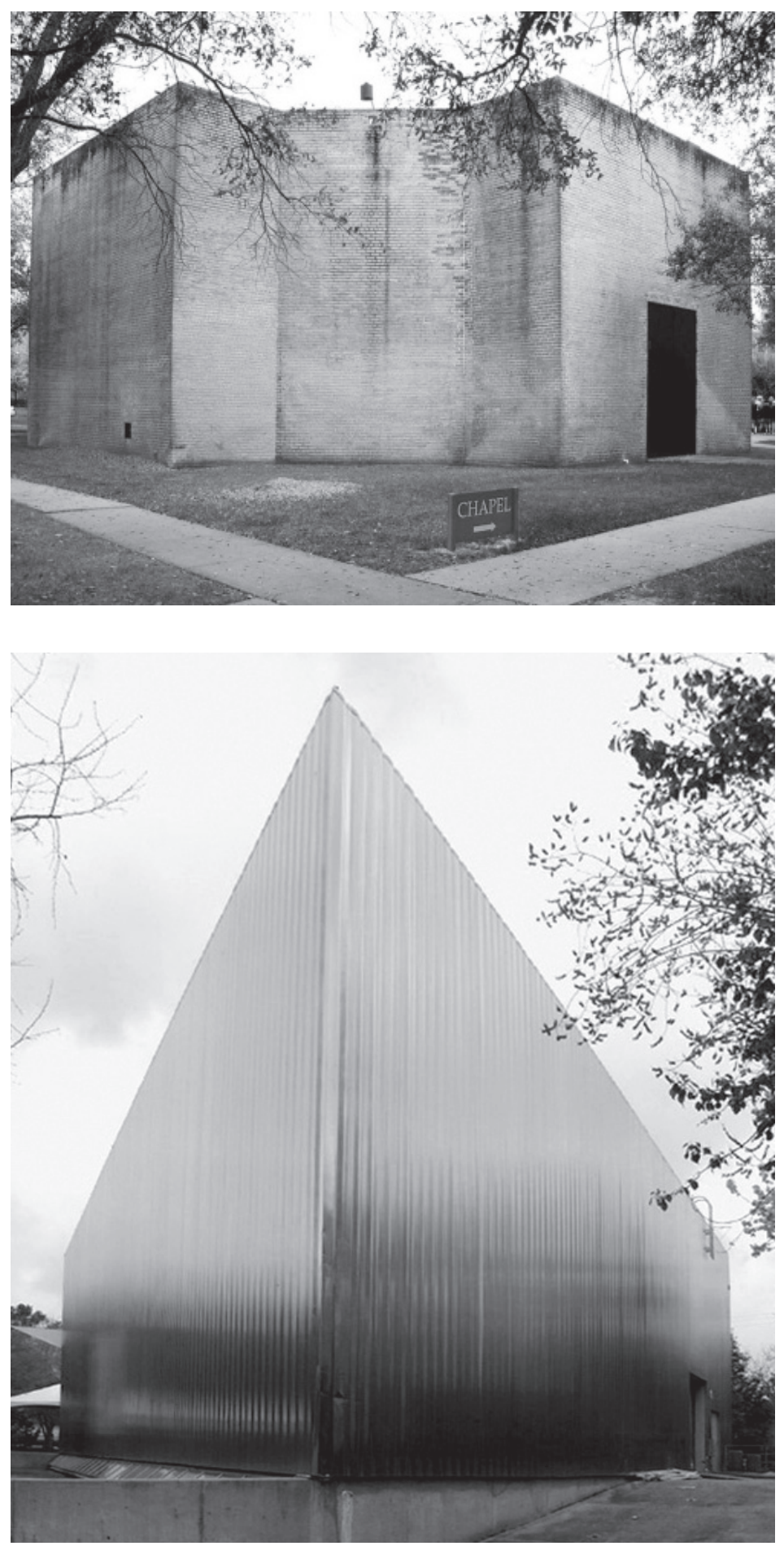

Fig. 2. Gunnar Birkits, Museo de Arte Contemporáneo, Houston, 1972. 


\begin{tabular}{|l|l|l|}
\hline \multicolumn{1}{|c|}{ CRITERIOS } & \multicolumn{1}{|c|}{ ROTHKO CHAPEL } & MUSEO DE ARTE CONTEMPORÁNEO \\
\hline 01. Tipo de lugar & $\begin{array}{l}\text { Lugar sagrado (institución sin ánimo de } \\
\text { lucro) }\end{array}$ & $\begin{array}{l}\text { Lugar secular (institución sin ánimo de } \\
\text { lucro) }\end{array}$ \\
\hline 02. Programa espacial & Espacio de galería & Espacio de galería \\
\hline 03. Denominación & Sin denominación & Sin denominación \\
\hline 04. Contexto del lugar & Urbano & Urbano \\
\hline 05. Contexto cultural & Houston, Texas & Houston, Texas \\
\hline 06. Vida del edificio & 1971 & 1972 \\
\hline 07. Proceso de diseño & $\begin{array}{l}\text { Diseñado por arquitectos profesionales } \\
\text { (Philip Johnson, Howard Barnstone, } \\
\text { Eugene Aubry) }\end{array}$ & $\begin{array}{l}\text { Diseñado por un arquitecto profesional } \\
\text { (Gunnar Birkits) }\end{array}$ \\
\hline 08. Tamaño & Superficie $=418,06 \mathrm{~m} 2$ & Superficie $=826,84 \mathrm{~m} 2$ \\
\hline 09. Visitas & Instalaciones abiertas todo el año & Instalaciones abiertas todo el año \\
\hline 10. Accesibilidad & $170 \mathrm{~km}$. de la localidad del investigador & $170 \mathrm{~km}$. de la localidad del investigador \\
\hline
\end{tabular}

TABLA 1. Análisis comparativo de criterios asociados a una selección de ejemplos a analizar

pueden discernirse y, en segundo lugar, pueden utilizarse para generar algo. Los patrones de creación de lugares, dentro del contexto de este estudio, pueden definirse como características espaciales y/o físicas que contribuyen a la creación de un sentido de lugar. Según Critchlow ${ }^{13}$, los patrones de creación de lugares expresan las intenciones y ejemplos más altos, e importantes valores culturales. Este estudio propone que estos patrones, si están presentes $\mathrm{y}$ - lo que es más importante- - si surgen en el lugar, tienen el potencial de transformar la arquitectura profana en un espacio sacro.

\section{METODOLOGÍA}

El objetivo del presente estudio es explorar las diferencias en la encarnación física de los patrones de creación de lugares entre la arquitectura sacra y la profana. Apoyándose en una serie de diez criterios (Tabla 1), los dos estudios de caso fueron la Rothko Chapel (Fig. 1) y el Museo de Arte Contemporáneo (Fig. 2), ambos ubicados en Houston, Texas. Se utilizaron estos criterios para conseguir una mayor conformidad entre los dos ejemplos con propósitos comparativos. La Rothko Chapel es un edificio sacro reconocido, y alberga un grupo de catorce pinturas de Mark Rothko. Las pinturas se exponen interiormente en la periferia de la planta octogonal. Además de exhibir la obra de Rothko, la capilla funciona como lugar de meditación privada, adoración común y celebración de coloquios relacionados con temas filosóficos y religiosos. Por otro lado, el Museo de Arte Contemporáneo es un edificio profano dedicado a exponer el arte contemporáneo.

La investigación fue realizada por medio de observaciones in situ, y conllevó un análisis arquitectónico de los dos edificios, sagrado y profano. Se realizaron múltiples visitas a ambas instalaciones. Se produjeron datos gráficos por medio de bocetos (con notas de campo) y fotografías de los dos edificios, sagrado y profano. Para la evaluación de las diferencias entre la encarnación física de los patrones de creación de lugares entre los dos edificios se realizó lo siguiente: en primer lugar, se identificaron las características físicas y espaciales de cada ubicación que constituyen el lugar; en segundo lugar, se compararon las características físicas y espaciales del edificio sacro con las características físicas y espaciales del edificio profano; y en tercer lugar, se identificaron las características físicas y espaciales que parecen únicas de cada escenario.

\section{RESULTADOS}

La Tabla 2 ofrece un resumen descriptivo de los patrones de creación de lugares individuales. Las características de cada escenario corresponden a temas subyacentes que aparecen en el libro de Christopher Alexander. Las figuras 3 y 4 muestran gráficamente cómo se encarnan dichos patrones dentro de cada sitio. 
ESTUDIO DE LAS DIFERENCIAS EN LA ENCARNACIÓN FÍSICA DE LOS PATRONES GENERADORES DE LUGAR...

\begin{tabular}{|c|c|}
\hline $\begin{array}{l}\text { PATRONES DE } \\
\text { CREACIÓN DE LUGARES }\end{array}$ & $\begin{array}{l}\text { RESUMEN DESCRIPTIVO DE LOS PATRONES } \\
\text { DE CREACIÓN DE LUGARES INDIVIDUALES }\end{array}$ \\
\hline Centro & $\begin{array}{l}\text { Punto focal o centro geométrico del lugar, habitualmente asociado a una actividad y } \\
\text { significado intensos. }\end{array}$ \\
\hline Envolvente & Cierres tales como paredes extriores, suelos, tejados del edificio, o borde del sitio. \\
\hline Dirección & $\begin{array}{l}\text { Alineación del edificio en el lugar, de modo que exista una orientación significativa } \\
\text { con los puntos cardinales. }\end{array}$ \\
\hline Descenso & $\begin{array}{l}\text { Características que ocupan el estrato inferior y permiten el descenso físico o visual, } \\
\text { tales como pisos bajos y entresuelos, cimientos, zapatas o pozos. }\end{array}$ \\
\hline Ascensión & $\begin{array}{l}\text { Características verticales que permiten el ascenso físico o visual, tales como torres, } \\
\text { líneas de la cubierta que ascienden verticalmente, columnas altas o haces de luz } \\
\text { desde arriba. }\end{array}$ \\
\hline Pasaje & $\begin{array}{l}\text { Puertas, vestíbulos, entradas o umbrales que ofrecen puntos de entrada al lugar. } \\
\text { Estos umbrales funcionan como claros espacios de continuidad entre dos ámbitos. }\end{array}$ \\
\hline Orden numérico & $\begin{array}{l}\text { Recurrencia significativa de características, tales como la singularidad o dualidad de } \\
\text { formas, el número de torres, puertas, ventanas, columnas, paredes o escalones. }\end{array}$ \\
\hline Orden geométrico & Formas que integran la forma física del edificio. \\
\hline Orden espacial & $\begin{array}{l}\text { Ritmo o sucesión de espacios que podrían revelar simetría, o ser circulares, lineales, } \\
\text { radiales, triangulares, ortogonales o espirales. }\end{array}$ \\
\hline Orden antropomórfico & Proporciones formales inspiradas en las medidas del cuerpo humano. \\
\hline Naturaleza ordenada & $\begin{array}{l}\text { Plantas, árboles y jardines especiales u otras características del paisaje natural que } \\
\text { están limitadas y controladas, es decir, cuidadas continuamente por las personas. }\end{array}$ \\
\hline Orden celestial & $\begin{array}{l}\text { Aperturas o marcas que indican el movimiento del sol, la luna u otros objetos } \\
\text { celestiales por medio de la alineación del edificio para articular solsticios. Puede } \\
\text { manifestarse por los techos abovedados. }\end{array}$ \\
\hline Límites diferenciadores & $\begin{array}{l}\text { Límites tales como paredes y tejados que revelan diferenciación, dependiendo de su } \\
\text { orientación con los puntos cardinales. }\end{array}$ \\
\hline Vistas ordenadas & $\begin{array}{l}\text { Aperturas limitadas o especialmente posicionadas que restringen y/o enmarcan las } \\
\text { vistas entre los correspondientes espacios para mejorar la experiencia dentro del } \\
\text { lugar. }\end{array}$ \\
\hline Materialidad & $\begin{array}{l}\text { Materiales de construcción, tales como ladrillo, piedra, madera, hormigón armado, } \\
\text { acero, baldosas cerámicas, escayola o vidrio. }\end{array}$ \\
\hline Elementos & $\begin{array}{l}\text { Fuego, agua, aire y tierra. Pueden expresarse de diversas formas, como chimeneas, } \\
\text { fuentes, aperturas que permiten la ventilación, o paredes sólidas de mampostería. }\end{array}$ \\
\hline Luz & $\begin{array}{l}\text { Luminosidad que puede orientar los puntos cardinales o marcar el paso del tiempo } \\
\text { con el movimiento del sol en el cielo. }\end{array}$ \\
\hline Orden Ceremonial & $\begin{array}{l}\text { Espacios que permiten la ceremonia, las celebraciones temporales/estacionales, la } \\
\text { oración, la meditación, o los actos rituales o de consagración en el lugar. }\end{array}$ \\
\hline
\end{tabular}

TABLA 2. Resumen descriptivo de los patrones de creación de lugares individuales 


\begin{tabular}{|c|c|c|}
\hline \multirow[t]{2}{*}{ PATRONES DE CREACIÓN DE LUGARES } & \multicolumn{2}{|c|}{ CARACTERÍSTICAS FÍSICAS Y ESPACIALES } \\
\hline & Rothko Chapel & Museo de Arte Contemporáneo \\
\hline Centro & $\begin{array}{l}\text { Centro geométrico de la planta } \\
\text { octogonal de la capilla }\end{array}$ & No se observó \\
\hline Envolvente & Paredes, suelo y techo de la capilla & Paredes, suelo y techo del museo \\
\hline Dirección & $\begin{array}{l}\text { Alineación de la entrada de la capilla } \\
\text { con un estanque al sur }\end{array}$ & No se observó \\
\hline Descenso & Oscuridad dentro de la capilla & Nivel bajo del museo \\
\hline Ascensión & $\begin{array}{l}\text { Luz que penetra por el lucernario del } \\
\text { techo de la capilla }\end{array}$ & No se observó \\
\hline Pasaje & Puerta de entrada principal de la capilla & Puerta de entrada principal del museo \\
\hline Orden numérico & Ocho lados de la capilla & No se observó \\
\hline Orden geométrico & Forma octogonal de la capilla & Paralelogramo del museo \\
\hline Orden espacial & $\begin{array}{l}\text { Transición jerárquica de espacios } \\
\text { desde el centro de la capilla al } \\
\text { estanque de afuera }\end{array}$ & No se observó \\
\hline Orden antropomórfico & Techos altos de la capilla & No se observó \\
\hline Naturaleza ordenada & $\begin{array}{l}\text { Estanque de agua con pantalla de } \\
\text { bambú }\end{array}$ & Matorrales in situ \\
\hline Orden celestial & Lucernario en el techo de la capilla & No se observó \\
\hline Límites diferenciadores & $\begin{array}{l}\text { Offsets variados en las paredes de la } \\
\text { capilla }\end{array}$ & No se observó \\
\hline Vistas ordenadas & $\begin{array}{l}\text { Las paredes de la capilla restringen la } \\
\text { vista al exterior }\end{array}$ & $\begin{array}{l}\text { Las paredes del museo restringen la } \\
\text { vista al exterior }\end{array}$ \\
\hline Materialidad & Paredes grises de la capilla & Lados metálicos del museo \\
\hline Elementos & $\begin{array}{l}\text { Paredes-tierra, lucernario-fuego, } \\
\text { estanque-agua, movimiento del } \\
\text { bosquecillo de bambú- viento }\end{array}$ & Fuente-agua \\
\hline Luz & $\begin{array}{l}\text { Luz solar que penetra por el lucernario } \\
\text { del techo y contrasta con la oscuridad } \\
\text { de la capilla }\end{array}$ & $\begin{array}{l}\text { Luz solar que penetra por las ventanas } \\
\text { de la planta baja del museo }\end{array}$ \\
\hline Orden ceremonial & $\begin{array}{l}\text { Solemne interacción de la gente con } \\
\text { las pinturas y con la capilla }\end{array}$ & No se observó \\
\hline
\end{tabular}

TABLA 3. Diferencias entre las características físicas y espaciales de los patrones de creación de lugares del edificio sagrado y el profano 
La evaluación de las características físicas y espaciales entre el edificio sacro y el profano, basada en observaciones realizadas en cada escenario, están resumidas en la Tabla 3.

\section{DISCUSIÓN}

Partiendo de los datos recopilados y analizados en la investigación, observe un total de nueve patrones de creación de lugares distintos y singulares en la Rothko Chapel comparada con el Museo de Arte Contemporáneo. Estos son: Centro, Dirección, Ascensión, Descenso, Orden numérico, Orden espacial, Orden antropomórfico, Orden celestial, Límites diferenciadores y Orden ceremonial. Los restantes nueve patrones (Envolvente, Descenso, Pasaje, Orden Geométrico, Naturaleza ordenada, Vistas ordenadas, Materialidad, Elementos y Luz) se hallaron comunes en ambos escenarios. El trabajo en curso especula, sin embargo, con que a pesar de que dichos patrones pueden ser comunes tanto en la arquitectura sacra como en la profana, la calidad de su expresión en el lugar podría tener un peso importante en su contribución a la sacralidad del lugar ${ }^{14}$. Los hallazgos de la investigación para este estudio y el actual trabajo en curso se utilizaron para desarrollar una matriz de patrones de creación de lugares (Fig. 5). La matriz intenta servir de guía para que los arquitectos creen lugares sacros.

La matriz se compone de dieciocho patrones de creación de lugares dispuestos en dos capas externas con Unidad en el centro de la matriz. Unidad significa la experiencia de todo o de integridad entre todos los patrones de la matriz. Denota la unificación integral y significativa de los dieciocho patrones que contribuyen a la sacralidad del lugar.

El trabajo actual en curso trata de la ordenación jerárquica de los patrones de creación de lugares en la matriz, y podría funcionar como indicador de la importancia de los patrones individuales en su contribución a la sacralidad del lugar. Es decir, es probable que la presencia y la calidad de la expresión de los patrones de creación de lugares en la capa intermedia de la matriz (en orden jerárquico descendente: Luz, Orden celestial, Centro, Orden ceremonial, Ascensión y Orden antropomórfico) tengan un mayor impacto en su contribución a la sacralidad del lugar que la presencia y la calidad de expresión de los restantes doce que se encuentran en la capa más externa de la matriz (en orden jerárquico descendente: Naturaleza ordenada, Vistas ordenadas,
Envolvente, Orden espacial, Elementos, Dirección, Orden numérico, Límites diferenciadores, Pasaje, Orden geométrico, Descenso y Materialidad). En este sentido, se especula con que la presencia y alta calidad de expresión del patrón de creación de lugares Luz puede que tenga una mayor influencia en su contribución a la sacralidad del lugar que la presencia y alta calidad de expresión del patrón de creación de lugares Materialidad.

\section{CONCLUSIÓN}

El estudio utiliza datos gráficos para explorar las diferencias en la encarnación física de los patrones de creación de lugares entre un edificio sacro (la Rothko Chapel) y uno profano (el Museo de Arte Contemporáneo). El contraste y la comparación entre los datos gráficos surgidos de los análisis arquitectónicos reveló que hay un total de nueve patrones (Centro, Dirección, Ascensión, Orden numérico, Orden espacial, Orden antropomórfico, Orden celestial, Límites diferenciadores, Orden ceremonial) que son distintivos y singulares en la Rothko Chapel, comparada con el Museo de Arte Contemporáneo.

Los restantes nueve (Envolvente, Descenso, Pasaje, Orden geométrico, Naturaleza ordenada, Vistas ordenadas, Materialidad, Elementos, Luz) se encontraron comunes a ambos escenarios.

Basándome en el trabajo actual en curso, especulo que la presencia y calidad de expresión de seis de los patrones de la capa intermedia de la matriz (Luz, Orden Celestial, Centro, Orden ceremonial, Ascensión y Orden antropomórfico) tienen más influencia en su contribución a la sacralidad del lugar que la presencia y calidad de expresión de los doce que se encuentran en la capa más exterior de la matriz (Naturaleza ordenada, Vistas ordenadas, Envolvente, Orden espacial, Elementos, Dirección, Orden numérico, Límites diferenciadores, Pasaje, Orden Geométrico, Descenso y Materialidad).

Serán necesarios más estudios para explorar cómo o de qué maneras influyen los patrones de creación de lugares de la matriz en la sacralidad del lugar. Otros estudios podrían comprobar también cómo la encarnación secuencial de los patrones contribuyen a la sacralidad del lugar. Finalmente, otros estudios podrían ayudar también a revelar las características y/o patrones que no han sido identificados en el presente trabajo.

A menudo se ha desprestigiado y malentendido la importancia de los patrones de creación de lugares. El 

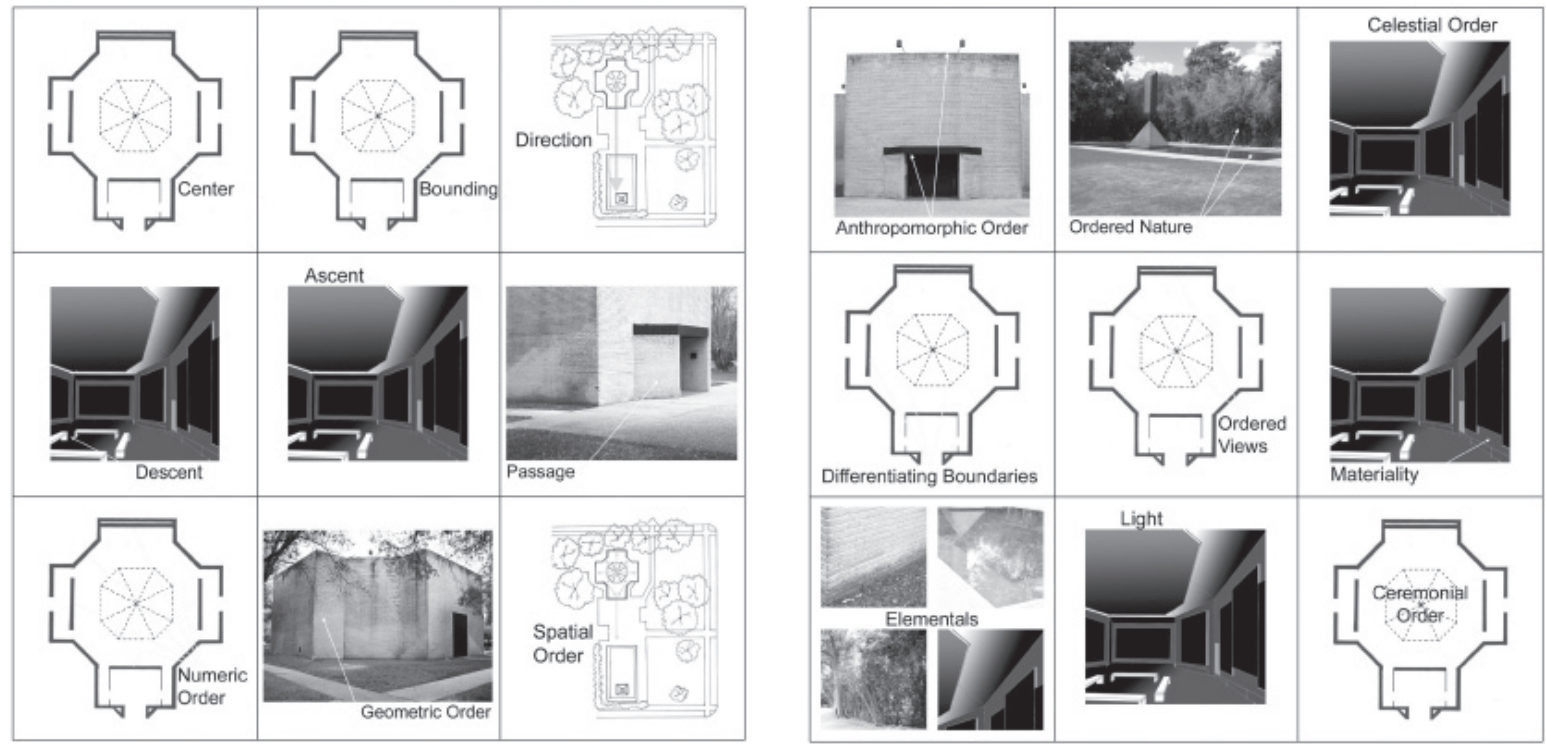

Fig. 3. Patrones de creación de lugares encarnados en la Rothko Chapel.

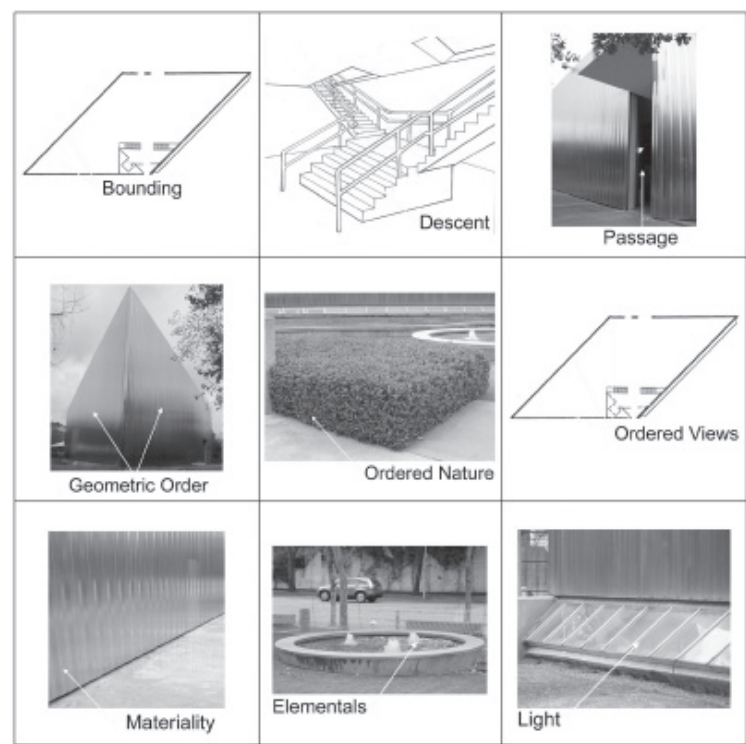

Fig. 4. Patrones de creación de lugares encarnados en el Museo de Arte Contemporáneo.

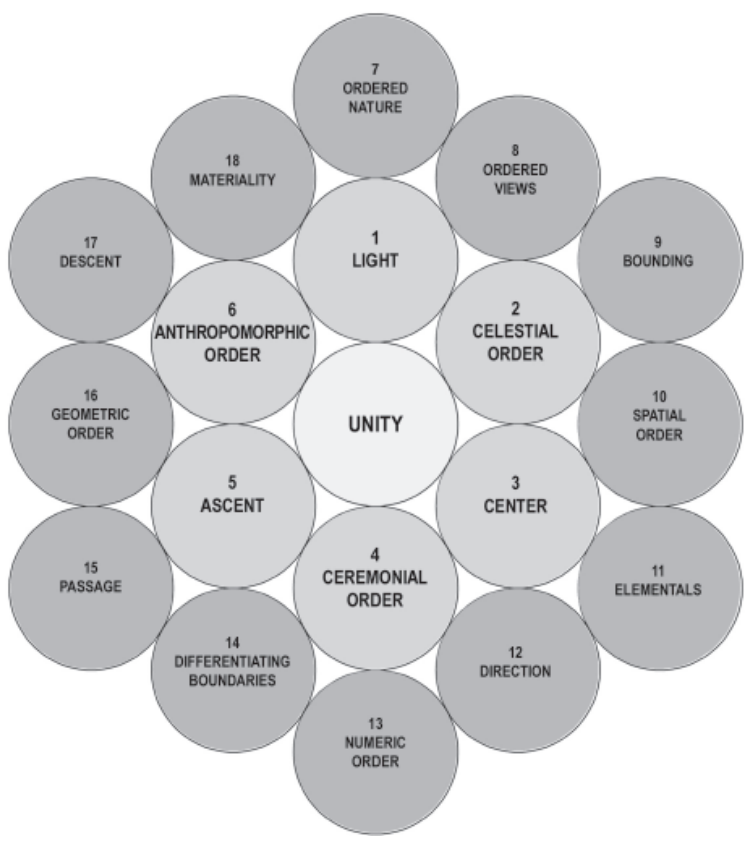

Fig. 5. Matriz de patrones de creación de lugares. 
contenido del presente texto puede verse como un pequeño paso en la re-evaluación del proceso, de los objetivos y del estatus de su uso en la actividad de diseño de lugares. Los patrones de creación de lugares, cuando son encarnados y elevados en un sitio de manera significativa, podrían servir de canales para la pura ejemplificación de lo sagrado. La arquitectura resultante podría, a su vez, servir de canal para recordar y experimentar lo sacro. A través de la lente de estos patrones se puede entender la naturaleza cualitativa del espacio sacro. En este sentido, los patrones podrían ayudar a crear un lugar que nos ayude a recordar nuestra búsqueda del modelo más ejemplar de lugar: aquél que es sagrado y que cura.

El camino es arduo y está lleno de peligros porque es, de hecho, un rito de paso de lo profano a lo sagrado, de lo efímero e ilusorio a la realidad y a la eternidad, de la muerte a la vida, del hombre a la divinidad. (Mircea Eliade, 1959)

\section{NOTAS}

(1) Cf. Anthony Lawlor, «The temple in the house: Finding the sacred in everyday architecture», Putnam's Sons, Nueva York, 1994.

(2) Cf. Robert C. Meurant, «The aesthetics of the sacred: A harmonic geometry of consciousness and philosophy of sacred architecture», The Opoutere Press, Whangamata, 1989.

(3) Mircea Eliade, «The sacred and the profane: The nature of religion», Harcourt, Nueva York, 1959; pág. 11.

(4) Loc. cit.

(5) Dover Publications, Nueva York, 1960.

(6) The MIT Press, Cambridge, 1997.

(7) Op. cit.

(8) Oxford University Press, Nueva York, 1977.

(9) Proceedings of the Council of Educators in Landscape Architecture's Annual Conference, Universidad de Illinois, Urbana, 1985; pág. 17-27.

(10) The MIT Press, Cambridge, 1994.

(11) The Academy for Sacred Architectural Studies, Denver, 1996.

(12) The Center for Environmental Structure, Berkeley, 2002.

(13) Cf. Keit Critchlow, «Lindisfarne letter 10, geometry and architecture: What is sacred in architecture», The Lindisfarne Association, West Stockbridge, 1980.

(14) Cf. Arsenio T. Rodrigues, «The Sacred in Architecture: A Study of the Presence and Quality of Place-making Patterns in Sacred and Secular Buildings», Tesis doctoral inédita, Texas University A\&M, College Station, 2008. 